\title{
Optimization of Superconducting Antenna arrays using RBF neural network
}

\author{
Ouarda Barkat ${ }^{1 \mathrm{a}}$, Abdelmadjid Benghalia ${ }^{1}$ \\ ${ }^{1}$ Electronics Department, University of Constantine, 25000 Constantine, Algeria
}

Received 10 December 2009, Accepted 15 February 2010

\begin{abstract}
In this work, RBF neural network is used to optimize the radiation pattern of non-uniform linear arrays of High superconducting rectangular microstrip antennas, on uniaxial anisotropic substrate. Using superconductors can reduce the insertion loss, and obtain a rather high gain. The full-wave method is used to computing the resonant frequency, and radiation pattern of superconducting rectangular antennas. Galerkin method is used in the resolution of the electric field integral equation. The radial basis function neural network is able to obtain feasible solutions, for the array by matching the desired $\mathrm{D}_{\mathrm{i}}$ and actual array patterns $F_{i}$. The results obtained from the simulation show that we reached our goals by a great degree of validity.
\end{abstract}

Key words: Superconducting rectangular, Antenna array, Full-wave, Radiation pattern, RBF Neura network

\section{Introduction}

In recent years, microstrip antennas play an increasingly significant role due to the advantages of their low profile, minimal weight, and cheap printed circuit construction.

In order to further improve the performance of patch antennas, it has been proposed to replace the conventional patch structures by superconductors. The recent successful results of High temperature superconducting thin film technology have been encouraged, in realization of microwave circuits and antennas. Major property of superconductor is very low surface resistance compared with normal metals, such as copper, silver, and gold. Using this low surface resistance $\mathrm{Z}_{\mathrm{s}}$, superconductors can reduce the insertion loss, and obtain a rather high gain, but suffers from a very narrow bandwidth, which severely limits its application [1], [2].

Large numbers of papers are devoted to the important problem of array synthesis. Recently, several microwave design optimization problems, are using, a stochastic technique of global optimization, based on genetic algorithms and Radial basis function (RBF) neural networks method [3]. This last method became increasingly popular thanks to its structural simplicity, and particularly, in design optimization of array antennas [3], [4].

\footnotetext{
${ }^{\mathrm{a}}$ Corresponding author: barkatwarda@yahoo.fr
}

In this paper a very efficient technique to derive the dyadic Green's function in the Fourier transform domain. The complex resonant frequency of a superconductor rectangular microstrip on uniaxial substrate is calculated, by using Galerkin method in solving the integral equation. The sinusoidal functions are selected as the basis functions, which show fast numerical convergence. The radiation pattern of non uniform linear arrays is presented by using the method of radial basis function neural network, which will be applied to the optimization of linear arrays of superconducting rectangular microstrip antennas on uniaxial anisotropic substrate.

\section{Formulation of problem}

As shown in Fig. 1, the geometry of non periodic array with a non uniform for even number of identical elements $(2 \mathrm{~N})$. The phase $\Omega_{\mathrm{i}}$, spacing $\mathrm{Y}_{\mathrm{i}}$ and current $\mathrm{I}_{\mathrm{i}}$, have symmetry with respect to the centre of the linear array.

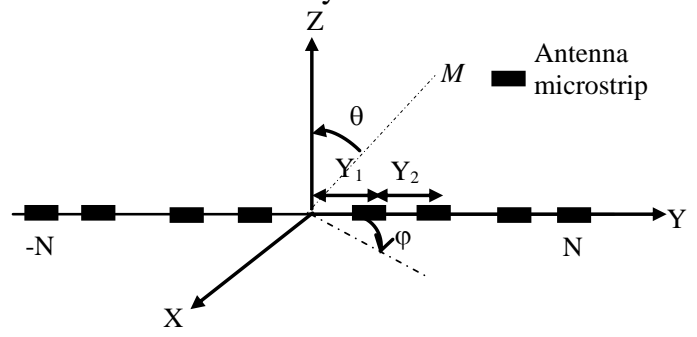

Fig. 1. Geometry of linear array of High superconducting rectangular microstrip antennas 
Each one element of the array antennas is a High superconducting rectangular microstrip as illustrated in fig.2, of thickness t, and dimension a, $\mathrm{b}$ along the two axes $\mathrm{y}, \mathrm{x}$, respectively, is printed on a grounded uniaxial dielectric of thickness $\mathrm{d}$.

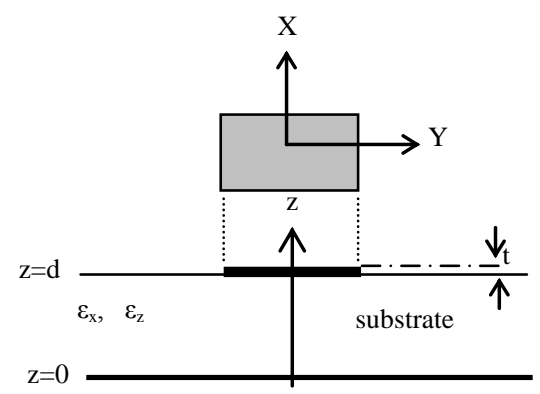

Fig. 2. Geometry of High superconducting rectangular microstrip patch on uniaxial substrate

The transverse field components in the $\mathrm{j}^{\text {th }}$ layer can be obtained using the Fourier transform formulation [5].

$$
\begin{aligned}
& \tilde{\bar{E}}\left(k_{s}, z\right)=\int_{-\infty-\infty}^{+\infty} \int_{-\infty} \bar{E}(x, y, z) \cdot e^{-j\left(k_{x} \cdot x+k_{y} \cdot y\right)} d x \cdot d y \\
& \tilde{\bar{H}}\left(k_{s}, z\right)=\int_{-\infty-\infty}^{+\infty+\infty} \int^{\infty} \bar{H}(x, y, z) \cdot e^{-j\left(k_{x} \cdot x+k_{y} \cdot y\right)} d x \cdot d y
\end{aligned}
$$

Starting from Maxwell's equations in the Fourier transform domain, the relationship between the patch current and the electric field on the patch is given by:

$$
\tilde{\bar{E}}\left(\mathbf{k}_{s}\right)=\bar{Q}\left(\mathbf{k}_{s}\right) \cdot \tilde{\bar{J}}\left(\mathbf{k}_{s}\right)
$$

Where $\bar{Q}$ is the spectral dyadic Green's function and $\widetilde{\bar{J}}\left(\mathbf{k}_{s}\right)$ is the current on the patch. In order to incorporate the finite thickness, the dyadic Green's function is modified by considering a complex boundary condition. The surface impedance of a high-temperature superconductors (HTS) material for a plane electromagnetic wave incident normally to its surface is defined as the ratio of $|\mathrm{E}|$ to $|\mathrm{H}|$ on the surface of the sample. It is given by:

$$
Z_{S}=R_{S}+j X_{S}
$$

Where $R_{s}$ and $X_{s}$ are the surface resistance and the surface reactance.

If the thickness ( $t$ ) of the strip of finite conductivity $\sigma$ is greater than three penetration depths, the surface impedance is adequately represented by the real part of the wave impedance.

$$
Z_{s}=\sqrt{\frac{\omega \mu_{0}}{2 . \sigma}}
$$

If the thickness of the superconducting microstrip (t) is less than three penetration depths, a better boundary condition is given by:

$$
Z_{s}=\frac{1}{t \sigma}
$$

Where the conductivity $\sigma=\sigma_{0}$ is real for conventional conductors. These approximations have been verified for practical metallization thicknesses, by comparison with rigorous mode matching result.

For superconductors, a complex conductivity of the form:

$$
\sigma=\sigma_{n}\left(T / T_{c}\right)^{4}-i\left(1-\left(T / T_{c}\right)^{4}\right) / \omega \mu_{0} \lambda_{0}^{2}
$$

where $\sigma_{\mathrm{n}}$ is often associated with the normal state conductivity at $T_{c}$ and $\lambda_{0}$ is the effective field penetration depth [2]. Now, we have the necessary Green's function, it is relatively straightforward to formulate the moment method solution for the antenna characteristics. The boundary condition at the surface of the high superconducting rectangular microstrip antennas is given by:

$$
\overline{\mathbf{E}}_{\text {scat }}+\overline{\mathbf{E}}_{\text {inc }}-\bar{Z}_{\mathrm{s}} \cdot \bar{J}=0
$$

Where $\overline{\mathrm{E}}_{\mathrm{scat}}$ and $\overline{\mathrm{E}}_{\mathrm{inc}}$ are tangential components of incident, scattered electrics fields, and $\bar{Z}_{s}$ is given by:

$$
\bar{Z}_{s}=\left(\begin{array}{cc}
Z_{s} & 0 \\
0 & Z_{s}
\end{array}\right)
$$

The transverse electric fields out of the patch can be expressed via the inverse Fourier transform as follows:

$$
\begin{aligned}
& E_{x}(x, y)=\frac{1}{4 \cdot \pi^{2}} \int_{-\infty}^{+\infty} \int_{-\infty}^{+\infty}\left[G_{x x}^{\prime}, \tilde{J}_{x}+G_{x y} \cdot \tilde{J}_{y}\right] e^{j\left(k_{x} \cdot x+k_{y} \cdot y\right)} \cdot d k_{x} \cdot d k_{y} \\
& E_{y}(x, y)=\frac{1}{4 \cdot \pi^{2}} \int_{-\infty}^{+\infty} \int_{-\infty}^{+\infty}\left[G_{y x} \tilde{J}_{x}+G_{y y}^{\prime} \cdot \tilde{J}_{y}\right] \cdot e^{j\left(k_{x} \cdot x+k_{y} \cdot y\right)} \cdot d k_{x} \cdot d k_{y} \\
& \text { where } G_{x x}^{\prime}=G_{x x}-Z_{s}, \text { and } G_{y y}^{\prime}=G_{y y}-Z_{s}
\end{aligned}
$$

The far zone radiation fields are derived using Huygens principle. The basic equations of far zone fields of High superconducting rectangular microstrip antennas are given as [5]:

$$
\begin{gathered}
E_{\phi}(\theta, \phi)=-E_{x} \sin \phi+E_{y} \cos \phi \\
E_{\theta}(\theta, \phi)=E_{x} \cos \theta \cos \phi+E_{y} \cos \theta \sin \phi
\end{gathered}
$$

In many array structures, High superconducting rectangular microstrip antennas find more applications than any other geometry of microstrip antenna. For simplicity in analysis, the overall radiation pattern of an antenna array can be 
obtained by multiplying the array factor, and the element pattern of the High superconducting rectangular antenna. Hence, in an array of identical antennas, the radiation pattern of the array is given by:

$$
A F(\theta, \phi)=\left(\left|\frac{E_{\theta, \Phi}}{E_{\max }}\right|\right) \cdot F(\theta, \phi)
$$

Where

$$
F(\theta, \phi)=\sum_{i=1}^{2 N} I_{i} \exp \left[j\left(k_{0} Y_{i} \sin \theta \cos \phi+\Omega_{i}\right)\right]
$$

Through, to determine the values of parameters $\left(\Omega_{\mathrm{i}}, \mathrm{Y}_{\mathrm{i}}\right.$ and $\left.\mathrm{I}_{\mathrm{i}}\right)$, we used the radial basis function (RBF) neural network to approximate the radiation pattern function, and the approximate optimum results, for getting better the radiation pattern of array. The radial basis function (RBF) neural network consists of three layers: the input layer where the inputs are applied, the hidden layer and the output layer. In our study, we have constructed the Radial basis function (RBF) network with nonlinear function, the most commonly used type of radial basis function is the Gaussian illustrated in Fig. 3. The procedure starts with a vector of inputs (x). Then the distance between the inputs and the vector of weights $\left(C_{j}\right)$ is calculated, and sent to the radial function.

The output of $j^{\text {th }}$ hidden neuron can be written as:

$$
h_{j}=e^{\left(-\frac{\left\|x-C_{j}\right\|^{2}}{\sigma_{j}^{2}}\right)}
$$

Where $\mathrm{x}$ is the input vector, $\mathrm{C}_{\mathrm{j}}$ is the neuron center, and $\sigma_{\mathrm{j}}$ is the center spread parameter. The neurons of the output layer have a linear transfer function, it is simply the weighted summation of outputs of all hidden neurons (gaussian RBF) connected to the output neuron. For the $\mathrm{k}^{\text {th }}$ neuron, the output $S_{k}$ is given by:

$$
S_{k}=\sum_{j=1}^{m} w_{k j} \cdot h_{j}
$$

Where $\mathrm{W}_{\mathrm{kj}}$ is the synaptic weight connecting hidden neuron $\mathrm{j}$ to output neuron $\mathrm{k}$, and $\mathrm{m}$ is the number of hidden layer neuron. The design of a $\mathrm{RBF}$ network is composed of two phases: the training process and the testing phase.

The training process is divided in two stages; Firstly, the parameters $C_{j}$ of the $R B F$ are determined by the k-means algorithm, secondly the optimal weight vector $\mathrm{W}_{\mathrm{kj}}$ is achieved using the least mean square (LMS) method.

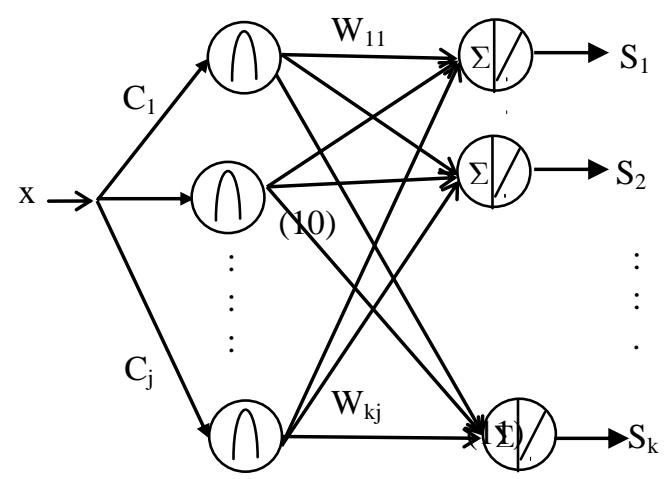

Fig. 3. RBF neural network architecture

Once the training phase is finished, we have generated several side lobe levels in order to validate the model gotten by the neural network.

\section{Results}

In this study, the proposed antenna microstrip has been simulated using the Galerkin method in the spectral domain. Figs. 4, 5 and 6 show the variation of normalized far field radiation patterns in plan $\mathrm{H}$, for the mode resonant frequency $(\mathrm{FR}=43.33 \mathrm{GHz})$ of the array of antennas, for three values of SLL $(-27 \mathrm{~dB},-39 \mathrm{~dB}$, and $-45 \mathrm{~dB})$.

The linear array considered in this study, containing 20 High superconducting rectangular microstrip, are printed on a substrate of permittivity $\varepsilon_{\mathrm{x}}=\varepsilon_{\mathrm{z}}=11.6$, with a thickness $\mathrm{d}=0.16 \mathrm{~b}$. The superconducting rectangular microstrip characteristics are: $\mathrm{a}=1630 \mu \mathrm{m}, \mathrm{b}=935 \mu \mathrm{m}$, $\sigma_{\mathrm{n}}=210 \mathrm{~s} / \mathrm{mm}, \lambda_{0}=1500 \AA, \mathrm{T} / \mathrm{T}_{\mathrm{c}}=0.5$ and $\mathrm{t}=0.02 \mu \mathrm{m}$.

In order to illustrate the capabilities of the Radial basis function (RBF) network, to find acceptable side lobe level (SLL), equal or less than the desired value, by varying elements excitations and positions, the (RBF) program has been written in Matlab language, the number of testing set is equal 80.

The far field radiation patterns obtained are compared with the far field radiation patterns by Dolph Chebyshev distribution. The value of beamwidth and side lobes (SLL) calculated is lower than the one find by Dolph Chebyshev distribution. 


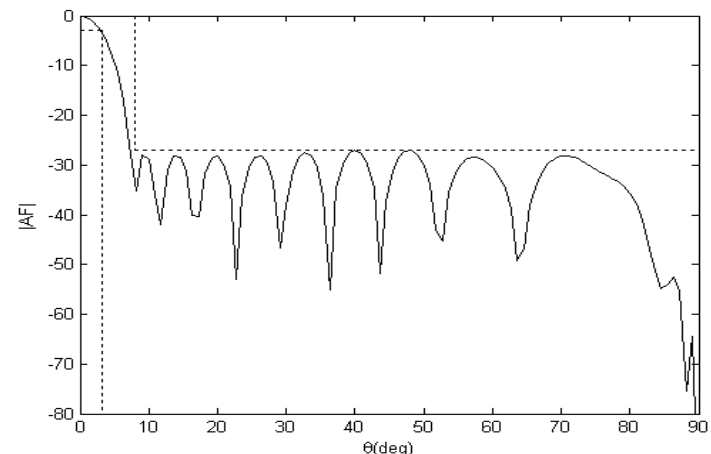

Fig. 4. Radiation patterns of linear array of 20 high superconducting rectangular microstrip antennas with:

$$
\text { SLL }=-27 d B
$$

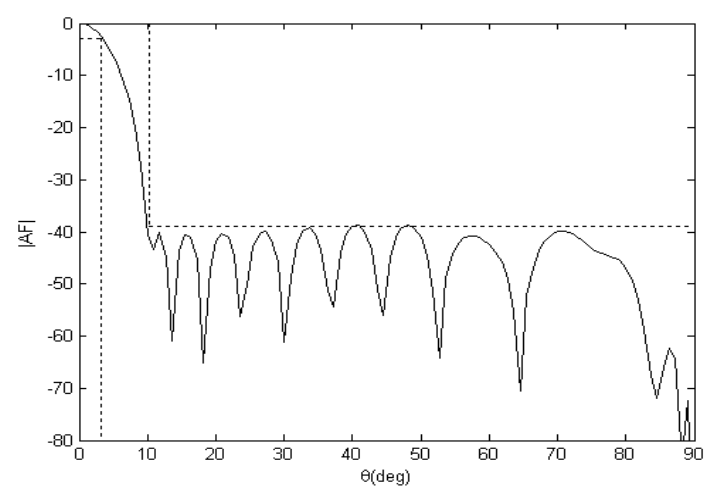

Fig. 5. Radiation patterns of linear array of 20 high superconducting rectangular microstrip antennas with: SLL $=-39 \mathrm{~dB}$

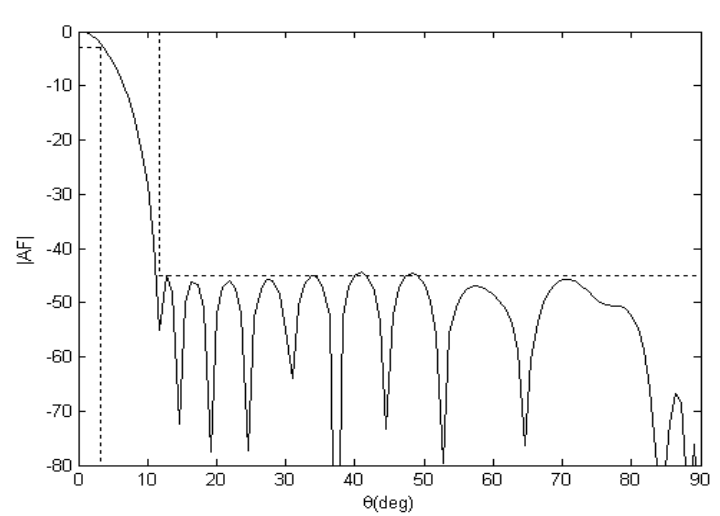

Fig. 6. Radiation patterns of linear array of 20 high superconducting rectangular microstrip antennas with: SLL $=-45 \mathrm{~dB}$

\section{Conclusion}

We have developed the radiation pattern of array of 20 High superconducting rectangular microstrip antennas, on uniaxial anisotropic substrate using the Galarkin method. The integral equation is formulated with vector Fourier. We have used a neural networks (RBF) in optimization of radiation pattern of non-uniform linear array. The results obtained by this approach are satisfactory, and show the interest of the application of the RBF in optimizing the performance of arrays while reducing the level of sidelobe. These results are important in the design of arrays with superconducting antennas.

\section{References}

1. R.C. Hansen, Electrically small, superdirective, and superconducting antennas ,John Wiley, Hoboken, New Jersey, (2006).

2. O. Barkat, A. Benghalia, Radiation and Resonant Frequency of Superconducting Annular Ring Microstrip Antenna on Uniaxial Anisotropic Media, Springer, Journal of Infrared, Millimeter and Terahertz Waves, 30, 1053-1066, (2009).

3. M. Sarevska, and A. B. M. Salem, Antenna array beamforming using neural network, Proceeding of World Academy of Science Engineering and Technology, 17, ISSN 1307-6884, 324-328, (2006).

4. V. K. Devabhaktuni, M. C. E. Yagoub, Y. Fang, J. $\mathrm{Xu}$, and Q. J. Zhang, Neural networks for microwave modelling: Model development issues and nonlinear modelling techniques, John Wiley \& Sons Inc, (2001).

5. W. Barkat, A. Benghalia, Radiation Pattern Synthesis for Linear Arrays of Microstrip antennas on Uniaxially Anistropic Substrate, IEEE International Conference on Computational Cybernetics, ISBN 078038379-6327-330, 209-213 (2007). 